\title{
A special issue on cell signaling, disease, and stem cells
}

\author{
Cell Research (2012) 22:1-2. doi:10.1038/cr.2012.7; published online 5 January 2012
}

As the basic unit of life, cells utilize signaling pathways to receive inputs from the environment and translate such information into appropriate cellular behaviors and responses. Cell signaling is also pivotal for multicellular organisms such as mammals, as cells need to communicate extensively among each other and with the environment in order to orchestrate appropriate actions, which are in turn integrated at the system level for the proper functioning and well-being of the organism. Thus, understanding the molecular mechanisms of cell signaling constitutes a fundamental quest of today's life science research. Not surprisingly, dysregulation of cell signaling causes many diseases such as cancer, and in such cases, a thorough understanding of the nature of cell signaling under disease states would provide an important basis to the efforts of developing novel therapeutic strategies. In this context, we are pleased to present this 2012 Cell Research Special Issue focusing on "Cell signaling, disease, and stem cells".

The first part of this special issue consists of 7 review articles covering diverse areas of research on cell signaling and disease. MAPK signaling is the prototype cellular signaling cascade. In the first of the 7 reviews, Melanie Cobb and colleagues discuss the role of a key upstream player, Raf, in the control of MAPK signaling under normal and disease conditions. Angiogenesis is essential for many physiological processes and also plays an important role in many pathological conditions. The review by Silvio Gutkind and colleagues describes our current understanding on how semaphorins and their receptors control normal and pathological angiogenesis. $\mathrm{T}$ cell response is a key element of adaptive immunity; in their article, Jiahuai Han and colleagues provide an updated synthesis of the knowledge on an intriguing type of $\mathrm{T}$ cell response, the public $\mathrm{T}$ cell response. Autophagy is a "self-eating" mechanism employed by cells to degrade various cellular components via the lysosomal pathway, and fulfills numerous functions in processes such as development, differentiation, and ageing. The article by Peter Vandenabeele and colleagues provides a comprehensive review on the autophgic signaling pathway, its role in normal cellular processes, and the potential contribution of autophay defects to disease. The rest 3 review articles also touch aspects of various diseases. Pablo Menendez and colleagues review the study of modeling sarcomagenesis using multipotent mesenchymal stem cells (MSCs) and discuss common mechanisms of MSC transformation. Hui Zheng and colleagues review the physiological and pathophysiological functions of amyloid precursor protein (APP), with a focus on insights gained from the analyses of various APP animal models. Jianming Xu and colleages review the normal and diseaserelated functions of Twist1, a transcription factor with well known roles in epithelial-mesenchymal transition and cancer metastasis.

The second part of the special issue is made of 12 original research articles. The large number of original research articles and the diverse nature of the topics covered by the review articles make this 2012 special issue distinct from many previous ones, such as the two issues on NF- $\mathrm{KB}$ and epigenetics/chromatin of last year [1, 2]. In the first research article, Chen$\mathrm{Yu}$ Zhang and colleagues report a landmark discovery of cross-kingdom regulation by miRNAs, whereby ricederived miR168a can enter mammals through food intake and regulate mammalian gene expression. In the second article, Quan Chen and colleagues elucidate a novel mechanism of feedback amplification of mitochondrial damage in apoptosis through caspase-mediate cleavage of cytochrome $\mathrm{c} 1$. The rest 10 original articles are all related to researches on stem cells. We chose to highlight these stem cell papers in this special issue as stem cell research is intrinsically connected to the study of cell signaling and disease. The collection of a large number of stem cell papers also reflects the fact that stem cell research has become an important part of the growing content of Cell Research, as highlighted by a large number of important discoveries published in the journal in the past year alone, such as the successful generation of iPS cells with a single factor Oct4 [3, 4], efficient generation of human iPS cells from blood cells via a non-integrating method [5], generation of functional platelets from hESCs [6], directed differentiation of atrial and ventricular myocytes from hESCs [7], the successful generation of ovine, hircine and bovine iPS cells [8-10], somatic cell reprogramming using electrofused blastomeres [11], improved methods for iPS cell generation $[12,13]$, gener- 
ating iPS cells using Bmi1 [14], comprehensive analyses of chromatin state dynamics in hESC differentiation [15], characterization of neuroblasts in the adult human brain [16], identification of lectin biomarkers for human pluripotent stem cell isolation [17], and efficient correction of disease-causing genetic mutations in patient iPS cells [18].

In keeping with this growing trend this special issue features 10 stem cell papers. Using an improved purification approach, Jianlong Wang and colleagues report the discovery of a comprehensive Oct4-linked pluripotency network in ES cells, identifying many novel Oct4 partners. Three papers are on iPS cells: Ken Chien and colleagues report the generation of iPS cells from murine ventricular myocytes and their subsequent highly efficient differentiation into ventricular cardiomyocytes; Juan Carlos Izpisua Belmonte and colleagues report the first global metabolome analysis of iPS cells; Huang-Tian Yang and colleagues show that ascorbic acid could enhance cardiac differentiation of iPS cells and explore the underlying mechanisms. The other side of the coin of stemness is differentiation, a topic covered by 4 papers in this issue. William Lowry and colleagues present comprehensive transcriptome profiling analyses of human pluripotent stem cell derivatives and their tissue-derived counterparts; Hongkui Deng and colleagues report a novel and improved stepwise strategy of generating hematopoietic progenitors from hESCs; Sangtaek Oh and colleagues report their identification of a novel small molecule agonist of the canonical Wnt pathway and its use in modulation of MSC differentiation; and finally, Qi Zhou and colleagues show the successful reprogramming of Sertoli cells into induced neural stem/ progenitor cells by defined factors, in a process called transdifferentiation.
The collection is completed with 2 papers on cancer stem cells (CSCs): Xianming Mo and colleagues report the first identification of CSCs from gastric adenocarcinoma patients; Yizheng Wang and colleagues present their identification of a specific population of tumor cells that play a critical role in hematogenous metastasis of liver cancers.

We hope that this collection of outstanding review and original articles will be a nice treat to our readers, and wish all of our readers and authors a happy 2012.

\section{Dangsheng $\mathrm{Li}^{1}$}

${ }^{1}$ Deputy Editor-in-Chief, Cell Research, Shanghai Institutes for Biological Sciences, Chinese Academy of Sciences, Shanghai 200031, China

dsli@sibs.ac.cn

\section{References}

1 Sun SC, Liu ZG. A special issue on $\mathrm{NF}-\kappa \mathrm{B}$ signaling and function. Cell Res 2011; 21:1-2.

2 Zhang Y. Recent progress in the epigenetics and chromatin field. Cell Res 2011; 21:373-374.

3 Li Y, Zhang Q, Yin X, et al. Generation of iPSCs from mouse fibroblasts with a single gene, Oct4, and small molecules. Cell Res 2011; 21:196-204.

4 Chen J, Liu J, Yang J, et al. BMPs functionally replace Klf4 and support efficient reprogramming of mouse fibroblasts by Oct 4 alone. Cell Res 2011; 21:205-212.

5 Chou BK, Mali P, Huang X, et al. Efficient human iPS cell derivation by a non-integrating plasmid from blood cells with unique epigenetic and gene expression signatures. Cell Res 2011; 21:518-529.

6 Lu SJ, Li F, Yin H, et al. Platelets generated from human embryonic stem cells are functional in vitro and in the microcirculation of living mice. Cell Res 2011; 21:530-545.

7 Zhang Q, Jiang J, Han P, et al. Direct differentiation of atrial and ventricular myocytes from human embryonic stem cells by alternating retinoid signals. Cell Res 2011; 21:579-587.

8 Bao L, He L, Chen J, et al. Reprogramming of ovine adult fibroblasts to pluripotency via drug-inducible expression of defined factors. Cell Res 2011; 21:600-608.

9 Ren J, Pak Y, He L, et al. Generation of hircine-induced pluripotent stem cells by somatic cell reprogramming. Cell Res 2011; 21:849-853.

10 Han X, Han J, Ding F, et al. Generation of induced pluripotent stem cells from bovine embryonic fibroblast cells. Cell Res 2011; 21:1509-1512.

11 Riaz A, Zhao X, Dai X, et al. Mouse cloning and somatic cell reprogramming using electrofused blastomeres. Cell Res 2011; 21:770-778.

12 Chen J, Liu J, Chen Y, et al. Rational optimization of reprogramming culture conditions for the generation of induced pluripotent stem cells with ultra-high efficiency and fast kinetics. Cell Res 2011; 21:884-896.

13 Wang Q, Xu X, Li J, et al. Lithium, an anti-psychotic drug, greatly enhances the generation of induced pluripotent stem cells. Cell Res 2011; 21:14241435.

14 Moon JH, Heo JS, Kim JS, et al. Reprogramming fibroblasts into induced pluripotent stem cells with Bmi1. Cell Res 2011; 21:1424-1435.

15 Hawkins RD, Hon GC, Yang C, et al. Dynamic chromatin states in human ES cells reveal potential regulatory sequences and genes involved in pluripotency. Cell Res 2011; 21:1393-1409.

16 Wang C, Liu F, Liu YY, et al. Identification and characterization of neuroblasts in the subventricular zone and rostral migratory stream of the adult human brain. Cell Res 2011; 21:1534-1550.

17 Wang YC, Nakagawa M, Garitaonandia I, et al. Specific lectin biomarkers for isolation of human pluripotent stem cells identified through array-based glycomic analysis. Cell Res 2011; 21:1551-1563.

18 Li M, Suzuki K, Qu J, et al. Efficient correction of hemoglobinopathy-causing mutations by homologous recombination in integration-free patient iPSCs. Cell Res 2011; 21:1740-1744. 